\title{
Validation of prone intraoperative measurements of global spinal alignment
}

\author{
Max Vaynrub, MD, Brandon P. Hirsch, MD, Jared Tishelman, BA, Dennis Vasquez-Montes, MS, \\ Aaron J. Buckland, MD, Thomas J. Errico, MD, and Themistocles S. Protopsaltis, MD
}

Hospital for Joint Diseases at NYU Langone Medical Center, New York, New York

\begin{abstract}
OBJECTIVE Verifying the adequacy of surgical correction of adult spinal sagittal deformity (SSD) leads to improved postoperative alignment and clinical outcomes. Traditionally, surgeons relied on intraoperative measurements of lumbar lordosis (LL) correction. However, T-1 pelvic angle (TPA) and its component angles more reliably predict postoperative alignment. While TPA is readily measured on standing radiographs, intraoperative radiographs offer poor resolution of the bicoxofemoral axis. A method to recreate this radiographic landmark by extrapolating preoperative measurements has been described. The authors aimed to assess the reliability of measurements of global spinal alignment obtained via geometrical reconstitution of the bicoxofemoral axis on prone intraoperative radiographs.
\end{abstract}

METHODS A retrospective review was performed. Twenty sets of preoperative standing full-length and intraoperative prone 36-inch lateral radiographs were analyzed. Pelvic incidence (PI) and sacral to bicoxofemoral axis distance (SBFD) were recorded on preoperative films. A perpendicular line was drawn on the intraoperative radiograph from the midpoint of the sacral endplate. This was used as one limb of the PI, and the second limb was digitally drawn at an angle that reproduced the preoperatively obtained $\mathrm{PI}$, extending for a distance that matched the preoperative SBFD. This final point marked the obscured bicoxofemoral axis. These landmarks were used to measure the L-1, T-9, T-4, and T-1 pelvic angles (LPA, T9PA, T4PA, and TPA, respectively) and LL. Two spine fellows and 2 attending spine surgeons made independent measurements and repeated the process in 1 month. Mixed-model 2-way intraclass correlation coefficient (ICC) and Cronbach's $\alpha$ values were calculated to assess interobserver, intraobserver, and scale reliability.

RESULTS Interobserver reliability was excellent for preoperative PI and intraoperative LPA, T9PA, and T4PA (ICC = $0.88,0.84,0.84$, and 0.93 , respectively), good for intraoperative TPA (ICC $=0.68$ ), and fair for preoperative SBFD (ICC $=0.60)$ and intraoperative LL (ICC $=0.50)$. Cronbach's $\alpha$ was $\geq 0.80$ for all measurements. Measuring PI on preoperative standing images had excellent intraobserver reliability for all raters $(I C C=0.89$, range $0.80-0.93)$. All raters but one showed excellent reliability for measuring the SBFD. Reliability for measuring prone LL was good for all raters (ICC = 0.71 , range $0.64-0.76$ ). The LPA demonstrated good to excellent reliability for each rater (ICC $=0.76$, range $0.65-0.81$ ). The thoracic pelvic angles tended to be more reliable at more distal vertebrae (T9PA ICC $=0.71$, range 0.49-0.81; T4PA ICC $=0.62$, range 0.43-0.83; TPA ICC $=0.56$, range 0.31-0.86).

CONCLUSIONS Intraoperative assessment of global spinal alignment with TPA and component angles is more reliable than intraoperative measurements of LL. Reconstruction of preoperatively measured PI and SBFD on intraoperative radiographs effectively overcomes poor visualization of the bicoxofemoral axis. This method is easily adopted and produces accurate and reliable prone intraoperative measures of global spinal alignment.

https://thejns.org/doi/abs/10.3171/2018.1.SPINE17808

KEYWORDS prone; radiograph; T-1 pelvic angle; TPA; L-1 pelvic angle; LPA; surgical technique

A DULT spinal sagittal deformity (SSD) is associated with significant disability and decreased quality of life. ${ }^{5}$ Surgical correction of SSD is known to correlate with improved functional outcomes and quality-of-life measures. The impact of surgery on sagittal parameters and clinical outcome has been demonstrated in studies focusing on the deleterious consequences of iatrogenic sagittal imbalance and the beneficial effects of surgical correction of SSD. ${ }^{4}$ Thus, attaining appropriate sagittal alignment in SSD surgery is a critical goal for maximizing patient outcome. ${ }^{20}$

ABBREVIATIONS ICC = intraclass correlation coefficient; $L L=$ lumbar lordosis; $L P A=L-1$ pelvic angle; $P I=$ pelvic incidence; $S B F D=$ sacral to bicoxofemoral axis distance; SSD = spinal sagittal deformity; T4PA = T-4 pelvic angle; T9PA = T-9 pelvic angle; TPA = T-1 pelvic angle.

SUBMITTED July 19, 2017. ACCEPTED January 2, 2018.

INCLUDE WHEN CITING Published online May 18, 2018; DOI: 10.3171/2018.1.SPINE17808. 
As the central role of sagittal spinal balance has become increasingly recognized, there has been a growing body of literature on the radiographic analysis of sagittal alignment. ${ }^{2}$ Spinopelvic and global spinal parameters have been extensively described, and a range of normative values has been published. ${ }^{21}$ Pelvic incidence (PI) is a stable morphological angle from which surgeons can infer the ideal lumbar lordosis (LL). Although PI-LL mismatch can accurately describe the lumbar contribution to deformity, it fails to describe more proximal changes. The T-1 pelvic angle (TPA) was introduced as a global measure of sagittal alignment, along with its component angles (T-4, T-9, and L-1 pelvic angles) that describe their respective spinal regions. 18,19

Preoperative radiographic planning involves the analysis of the aforementioned parameters on standing fulllength radiographs. ${ }^{13}$ The deviations from normative values are calculated and used to identify the surgical goals of correction. ${ }^{16}$ Mathematical formulas have been described to predict postoperative alignment based on osteotomies planned on preoperative films, but such analyses are complex and cumbersome and a certain degree of error exists. ${ }^{9,22}$ Therefore, it is imperative to assess the changes in alignment after prone positioning ${ }^{6,10}$ and after corrective surgical maneuvers have been conducted. ${ }^{15}$ Intraoperative long-cassette prone radiography allows the deformity surgeon to verify that the alignment parameters have been attained, and if not, to make real-time adjustments as necessary. This method has been shown to accurately predict immediate postoperative alignment in scoliosis surgery. ${ }^{11}$

Traditionally, intraoperative prone measurements have focused on assessing LL and PI-LL mismatch. ${ }^{12}$ However, a prior study has shown that intraoperative measurements of TPA and component angles more reliably predict postoperative alignment than PI-LL. ${ }^{17}$ Surgeons often defer these global alignment measurements intraoperatively because the bicoxofemoral axis (a required landmark for the thoracic pelvic angles) is poorly visualized. A novel technique to recreate the bicoxofemoral axis via geometrical extrapolation has recently been described and promises to overcome this hurdle. ${ }^{17}$ The authors reported superior correlation of intraoperative TPA, T4PA, and T9PA, as compared with intraoperative PI-LL, with postoperative sagittal alignment.

The purpose of this study was to validate the intraoperative measurements of recently described parameters of global sagittal alignment (TPA, T4PA, T9PA, and LPA) on prone intraoperative full-spine radiographs. We aim to describe the interobserver and intraobserver reliability of this novel method.

\section{Methods \\ Study Criteria}

This is a retrospective review of a prospectively gathered database of patients with full-body standing lateral radiographs from a single institution. Approval of the IRB was obtained. We analyzed the records of 20 randomly selected patients from a list of patients undergoing operative correction of a minimum of 4 vertebral levels for sagittal deformity. Inclusion criteria consisted of patients with
SSD who underwent corrective surgery and had adequate preoperative and intraoperative radiographs. Patients with prior instrumentation undergoing revision surgery were included. Preoperative imaging included standing lateral 36-inch radiographs or full-body stereotactic radiographs (EOS Imaging). The preoperative radiographs required adequate visualization of T1, the sacral endplate, and bicoxofemoral axis. Intraoperative radiographs were prone lateral 36-inch films and required visualization of the sacral endplate and, at a minimum, the L-1 and T-9 vertebral bodies. For radiographs in which T-4 and T-1 were not identifiable, an estimate of their locations was made by the observer, and this was used to obtain measurements of T4PA and TPA. Visualization of the bicoxofemoral axis was not required for the intraoperative imaging.

\section{Data Collection}

Two orthopedic spine fellows (M.V., B.P.H.) and 2 attending orthopedic spine surgeons (A.J.B., T.S.P.) made independent measurements. The order of the patients was then randomly rearranged and the process was repeated by each observer at least 1 month later. All measurements were performed digitally using the institution's Picture Archiving and Communication System (PACS) software. Linear measurements on the preoperative films used the default calibration. Calibration was then equalized between preoperative and intraoperative imaging by using an anatomical landmark (such as the width of a vertebral body) or prior instrumentation (such as a known rod diameter) that was clearly visible on both films.

Pelvic incidence (PI) and the distance from the midpoint of the sacral endplate to the bicoxofemoral axis (sacral to bicoxofemoral axis distance [SBFD]) were recorded on preoperative films (Fig. 1 left). After identifying the sacral endplate on the intraoperative film, a perpendicular line was drawn from its midpoint. This was then used as one limb of the PI, and the second limb was digitally drawn at an angle that reproduced the preoperatively measured PI. The second limb was extended for a distance that matched the preoperative SBFD, and this final point marked the obscured bicoxofemoral axis (Fig. 1 right). These landmarks were then used to measure the TPA and its component angles: L-1 pelvic angle (LPA), T-9 pelvic angle (T9PA), and T-4 pelvic angle (T4PA; Fig. 2). The observers also measured the LL.

\section{Statistical Analysis}

Mixed-model 2-way intraclass correlation coefficient (ICC) was calculated to assess interobserver and intraobserver reliability. ICCs are used to assess for consistency and reproducibility of the radiographic measurements made by the various observers. ICCs were assessed using the following ranges: excellent $(0.75-1.0)$, good $(0.60-0.74)$, fair $(0.40-0.59)$, and poor $(<0.40)$. Cronbach's $\alpha$ is used to determine the extent of internal consistency. All statistical analysis was performed using the Statistical Package for the Social Sciences (SPSS version 23.0, IBM Corp.).

\section{Results}

Twenty patients with complete preoperative and intra- 
M. Vaynrub et al.

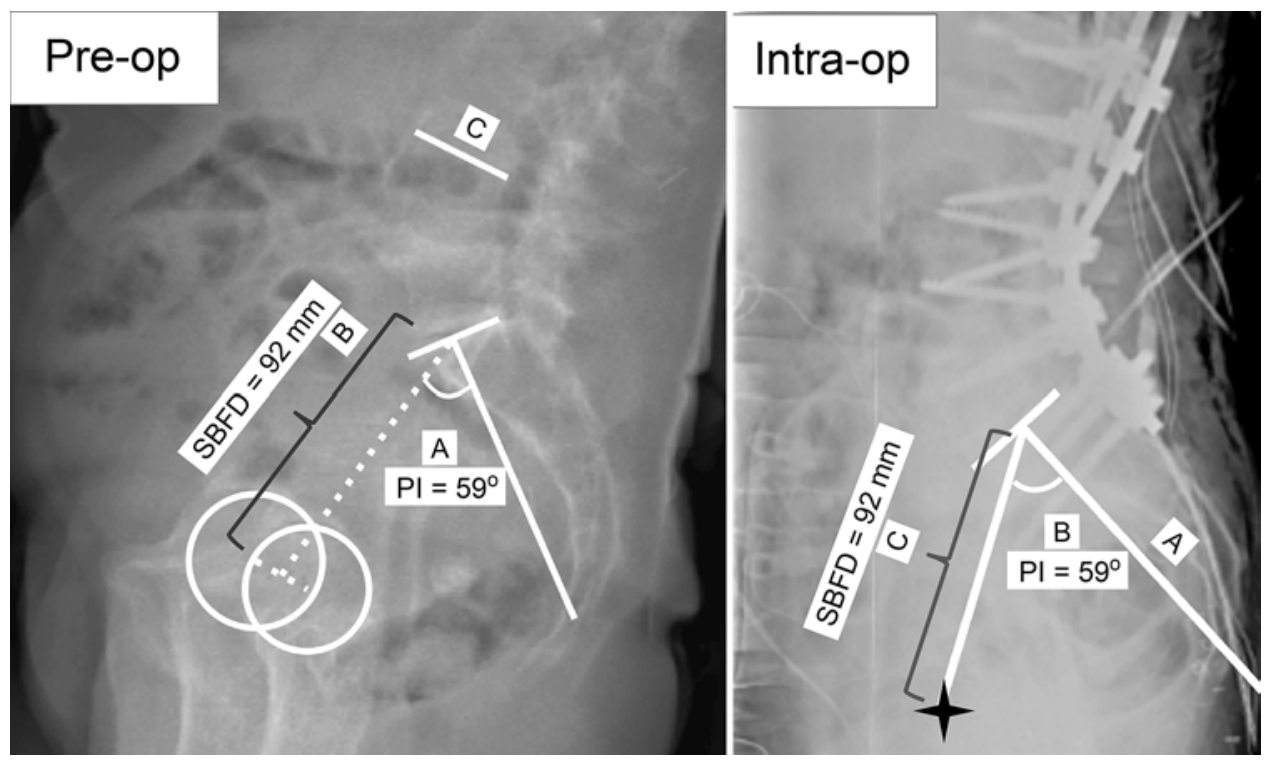

FIG. 1. Methodology for verifying the femoral head position on intraoperative radiographs based on preoperative imaging. Left: $A$ preoperative radiograph is analyzed first. PI is measured (A), SBFD is obtained (B), and length of easily identified anatomical structure is measured $(C)$. This is then used to calibrate the scale of the intraoperative image using the same anatomical structure (not shown). Right: The intraoperative radiograph is analyzed. Sacral endplate and a line perpendicular to its midpoint are drawn (A). The first limb of the PI is shown (B). The second limb of the PI is recreated using the preoperatively measured angle (C). The second limb is extended for a distance equal to preoperatively obtained SBFD. The end of this limb represents the recreated bicoxofemoral axis (star).
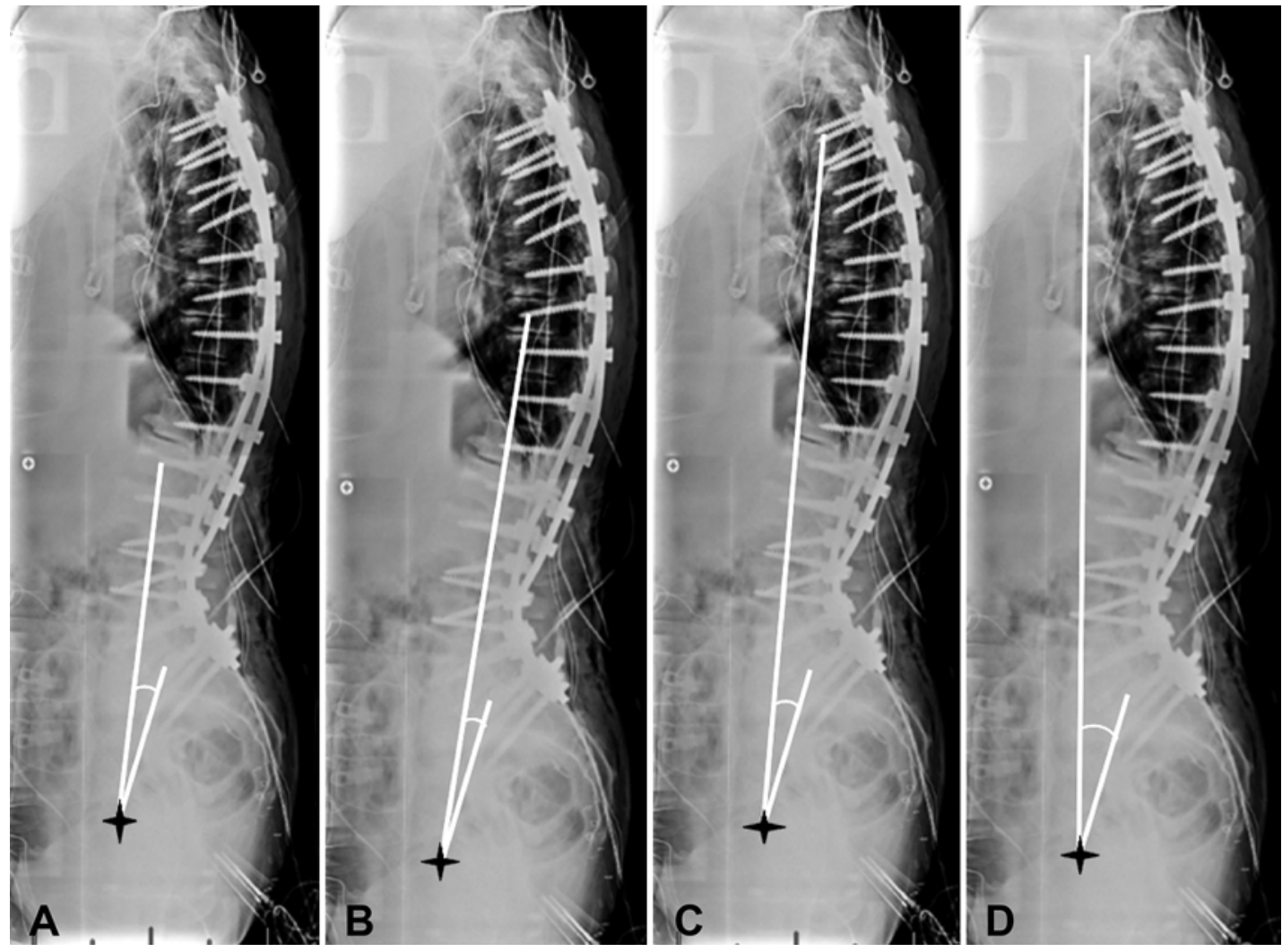

FIG. 2. Global sagittal parameters projected on intraoperative radiograph: LPA (A), T9PA (B), T4PA (C), and TPA (D). 
TABLE 1. Mean, median, and range of global measurements

\begin{tabular}{cccc}
\hline Measurement & Mean & Median & Range \\
\hline LPA & $18^{\circ}$ & $16^{\circ}$ & $1^{\circ}-43^{\circ}$ \\
\hline T9PA & $16^{\circ}$ & $14^{\circ}$ & $0^{\circ}-43^{\circ}$ \\
\hline T4PA & $18^{\circ}$ & $18^{\circ}$ & $0^{\circ}-42^{\circ}$ \\
\hline TPA & $21^{\circ}$ & $21^{\circ}$ & $1^{\circ}-46^{\circ}$ \\
\hline
\end{tabular}

operative radiographs were included for analysis. We report the mean, median, and range of values for the global sagittal parameters (TPA, T4PA, T9PA, LPA) in this series of patients (Table 1). Interobserver reliability was excellent for preoperative PI and intraoperative LPA, T9PA, and T4PA (ICC $=0.88,0.84,0.84$, and 0.93 , respectively), good for intraoperative TPA (ICC $=0.68$ ), and fair for preoperative SBFD and intraoperative LL (0.60 and 0.50, respectively). Cronbach's $\alpha$ was $\geq 0.80$ for all measurements (Table 2).

Measuring PI on preoperative standing images had excellent intraobserver reliability for all raters $(\mathrm{ICC}=0.89$, range $0.80-0.93)$. All raters but one showed excellent reliability for measuring the SBFD (Table 3). Reliability for measuring prone LL was good for all raters $(\mathrm{ICC}=0.71$, range 0.64-0.76). The LPA demonstrated good to excellent reliability for each rater $(\mathrm{ICC}=0.76$, range $0.65-0.81$ ). The thoracic pelvic angles trended to be more reliable at more distal vertebrae (T9PA ICC $=0.71$, range $0.49-0.81$; T4PA ICC $=0.62$, range $0.43-0.83$; TPA ICC $=0.56$, range $0.31-0.86)$. Interestingly, intraobserver reliability did not appear to be vary significantly with the surgical experience level of the rater.

\section{Discussion}

Reconciliation of postoperative alignment based on the preoperative plan is imperfect. ${ }^{1}$ Postoperative alignment is affected by several variables, including patient age, degree of intraoperative correction achieved, flexibility of unfused segments, and tendency of these segments to undergo reciprocal changes that counteracts the surgical correction (e.g., increased postoperative thoracic kyphosis proximal to the upper instrumented vertebra). ${ }^{3,7,8}$ Intraoperative prone radiography provides the closest estimation of postoperative alignment. Because fusion to the upper thoracic region mitigates the variable of postoperative thoracic compensation, it is associated with more reliable correlation between intraoperative and postoperative imaging. ${ }^{17}$

Traditional prone intraoperative radiographic measurements of lumbar lordosis fail to take thoracic alignment into account. In contrast, prone measurements of global sagittal alignment (i.e., TPA and its component angles) incorporate thoracic alignment and therefore more accurately predict postoperative global alignment. ${ }^{17}$ Radiographic identification of the femoral heads and the sacral endplate are required in obtaining global measurements. However, adequate lateral pelvic imaging is often difficult to obtain intraoperatively, particularly in obese patients. ${ }^{14}$ The present study validates a previously described method to recreate the bicoxofemoral axis on intraoperative radiographs based on preoperative radiographs. ${ }^{17}$
TABLE 2. Interobserver reliability

\begin{tabular}{ccc}
\hline Measurement & ICC & Cronbach's $\alpha$ \\
\hline PI & 0.88 & 0.89 \\
\hline LL & 0.50 & 0.80 \\
\hline SBFD & 0.60 & 0.86 \\
\hline LPA & 0.84 & 0.96 \\
\hline T9PA & 0.84 & 0.96 \\
\hline T4PA & 0.93 & 0.93 \\
\hline TPA & 0.68 & 0.89 \\
\hline
\end{tabular}

Of the multiple variables measured, intraoperative LL demonstrated the lowest interobserver reliability and internal consistency. This is not surprising, considering the type of observations required to produce this angle. The orientations of the sacral endplate and L-1 superior endplate must be identified. Although identification of the sacral endplate is a source of error that is common to both LL and the TPA component angles, identification of the orientation of the L-1 superior endplate is a challenge that is specific to LL. Although the L-1 vertebral body is relatively unobscured, coronal deformity complicates measurements of endplate orientation and introduces error to LL values. In contrast, measurement of TPA and its component angles relies on identification of the centroid of the vertebral bodies, an anatomical landmark that is usually straightforward to identify, even in the face of coronal deformity.

Measurements of global spinal alignment tended to have decreased intraobserver reliability in the upper thoracic spine (TPA and T4PA), as compared with T9PA and LPA. Similarly, although TPA had good interobserver reliability, it was lower than the more distal component angles. Measurements in the upper thoracic spine were complicated by two factors: inadequate penetration of T-1 due to radiographic interference from the shoulders, and inadequate length or positioning of the intraoperative cassette, truncating the image below the desired level. Despite these limitations, the observers were able to estimate the location of the upper thoracic vertebral bodies while maintaining good to excellent intraobserver and interobserver reliability.

One attribute of a desirable measurement system is ease of adoption. Although the attending surgeons in this study had been using the described method of prone intraoperative measurements for approximately 12 months, the fellows only gained exposure to this technique at the beginning of the study. In addition, the attending surgeons had significantly more practice with identifying landmarks and making radiographic measurements in general. Despite this considerable difference in experience levels, we did not observe a significantly better intraobserver reliability among the attending surgeons, suggesting that proficiency in this method is rapidly acquired.

There are several limitations to this study. We used 36-inch digitally processed cassettes for intraoperative radiography, limiting the generalizability of our results to centers with equivalent or similar capabilities. Conversely, many of our intraoperative films were suboptimally pen- 
TABLE 3. Intraobserver reliability for all 4 raters

\begin{tabular}{|c|c|c|c|c|c|c|c|c|c|c|}
\hline \multirow[b]{2}{*}{ Measurement } & \multicolumn{2}{|c|}{ Rater 1} & \multicolumn{2}{|c|}{ Rater 2} & \multicolumn{2}{|c|}{ Rater 3} & \multicolumn{2}{|c|}{ Rater 4} & \multicolumn{2}{|c|}{ Mean (Range) } \\
\hline & ICC & $\alpha$ & ICC & $\alpha$ & ICC & $\alpha$ & ICC & $\alpha$ & ICC & $\alpha$ \\
\hline $\mathrm{PI}$ & 0.93 & 0.96 & 0.91 & 0.95 & 0.92 & 0.96 & 0.80 & 0.89 & $0.89(0.80-0.93)$ & $0.94(0.89-0.96)$ \\
\hline LL & 0.76 & 0.92 & 0.64 & 0.78 & 0.71 & 0.83 & 0.71 & 0.83 & $0.71(0.64-0.76)$ & $0.84(0.78-0.92)$ \\
\hline SBFD & 0.88 & 0.94 & 0.88 & 0.94 & 0.34 & 0.51 & 0.86 & 0.92 & $0.74(0.34-0.88)$ & $0.83(0.51-0.94)$ \\
\hline LPA & 0.81 & 0.90 & 0.79 & 0.88 & 0.80 & 0.89 & 0.65 & 0.79 & $0.76(0.65-0.81)$ & $0.86(0.79-0.90)$ \\
\hline T9PA & 0.81 & 0.90 & 0.72 & 0.84 & 0.80 & 0.89 & 0.49 & 0.66 & $0.71(0.49-0.81)$ & $0.82(0.66-0.90)$ \\
\hline T4PA & 0.83 & 0.91 & 0.63 & 0.77 & 0.60 & 0.75 & 0.43 & 0.60 & $0.62(0.43-0.83)$ & $0.76(0.60-0.91)$ \\
\hline TPA & 0.86 & 0.92 & 0.68 & 0.81 & 0.40 & 0.57 & 0.31 & 0.47 & $0.56(0.31-0.86)$ & $0.69(0.47-0.92)$ \\
\hline
\end{tabular}

etrated or positioned, owing to the constraints of intraoperative imaging. Improved radiographic techniques could alter the relative reliabilities of our measured parameters. Finally, although intraoperative global alignment measurements are more predictive of postoperative alignment than are measurements of LL, additional factors such as postoperative compensatory changes must still be taken into account.

\section{Conclusions}

Intraoperative assessment of global spinal alignment with TPA and component angles is preferable to intraoperative assessment of LL for predicting postoperative alignment. Reconstruction of preoperatively measured PI and SBFD on intraoperative radiographs effectively overcomes poor visualization of the bicoxofemoral axis. This method is easily adopted and produces accurate and reliable prone intraoperative measures of global spinal alignment.

\section{References}

1. Ailon T, Scheer JK, Lafage V, Schwab FJ, Klineberg E, Sciubba DM, et al: Adult spinal deformity surgeons are unable to accurately predict postoperative spinal alignment using clinical judgment alone. Spine Deform 4:323-329, 2016

2. Bess S, Protopsaltis TS, Lafage V, Lafage R, Ames CP, Errico T, et al: Clinical and radiographic evaluation of adult spinal deformity. Clin Spine Surg 29:6-16, 2016

3. Day LM, Ramchandran S, Jalai CM, Diebo BG, Liabaud B, Lafage R, et al: Thoracolumbar realignment surgery results in simultaneous reciprocal changes in lower extremities and cervical spine. Spine (Phila Pa 1976) 42:799-807, 2017

4. Glassman SD, Berven S, Bridwell K, Horton W, Dimar JR: Correlation of radiographic parameters and clinical symptoms in adult scoliosis. Spine (Phila Pa 1976) 30:682-688, 2005

5. Glassman SD, Bridwell K, Dimar JR, Horton W, Berven S, Schwab F: The impact of positive sagittal balance in adult spinal deformity. Spine (Phila Pa 1976) 30:2024-2029, 2005

6. Harimaya K, Lenke LG, Mishiro T, Bridwell KH, Koester LA, Sides BA: Increasing lumbar lordosis of adult spinal deformity patients via intraoperative prone positioning. Spine (Phila Pa 1976) 34:2406-2412, 2009

7. Lafage R, Schwab F, Glassman S, Bess S, Harris B, Sheer J, et al: Age-adjusted alignment goals have the potential to reduce PJK. Spine (Phila Pa 1976) 42:1275-1282, 2017

8. Lafage V, Ames C, Schwab F, Klineberg E, Akbarnia B,
Smith J, et al: Changes in thoracic kyphosis negatively impact sagittal alignment after lumbar pedicle subtraction osteotomy: a comprehensive radiographic analysis. Spine (Phila Pa 1976) 37:E180-E187, 2012

9. Lafage V, Schwab F, Vira S, Patel A, Ungar B, Farcy JP: Spino-pelvic parameters after surgery can be predicted: a preliminary formula and validation of standing alignment. Spine (Phila Pa 1976) 36:1037-1045, 2011

10. Lee SK, Lee SH, Song KS, Park BM, Lim SY, Jang G, et al: Lumbar lordosis of spinal stenosis patients during intraoperative prone positioning. Clin Orthop Surg 8:65-70, 2016

11. Lehman RA Jr, Lenke LG, Helgeson MD, Eckel TT, Keeler KA: Do intraoperative radiographs in scoliosis surgery reflect radiographic result? Clin Orthop Relat Res 468:679686,2010

12. Leveque JC, Edwards A, Sethi RK: Preoperative, intraoperative, and postoperative standing lordosis after pedicle subtraction osteotomy: an analysis of radiographic parameters and surgical strategy. Spine Deform 4:245-250, 2016

13. Maggio D, Ailon TT, Smith JS, Shaffrey CI, Lafage V, Schwab F, et al: Assessment of impact of standing longcassette radiographs on surgical planning for lumbar pathology: an international survey of spine surgeons. J Neurosurg Spine 23:1-8, 2015

14. Miller AN, Krieg JC, Chip Routt ML Jr: Lateral sacral imaging in the morbidly obese. J Orthop Trauma 27:e122-e124, 2013

15. Moal B, Schwab F, Ames CP, Smith JS, Ryan D, Mummaneni PV, et al: Radiographic outcomes of adult spinal deformity correction: a critical analysis of variability and failures across deformity patterns. Spine Deform 2:219-225, 2014

16. Ondra SL, Marzouk S, Koski T, Silva F, Salehi S: Mathematical calculation of pedicle subtraction osteotomy size to allow precision correction of fixed sagittal deformity. Spine (Phila Pa 1976) 31:E973-E979, 2006

17. Oren JH, Day LM, Baker JF, Foster NA, Moses MJ, Ramchandran S, et al: Measurement of spinopelvic angles on prone intraoperative long-cassette lateral radiographs predicts postoperative standing global alignment in adult spinal deformity surgery. Spine J 16:S278, 2016 (Abstract)

18. Protopsaltis T, Schwab F, Bronsard N, Smith JS, Klineberg $\mathrm{E}$, Mundis $\mathrm{G}$, et al: The T1 pelvic angle, a novel radiographic measure of global sagittal deformity, accounts for both spinal inclination and pelvic tilt and correlates with health-related quality of life. J Bone Joint Surg Am 96:1631-1640, 2014

19. Ryan DJ, Protopsaltis TS, Ames CP, Hostin R, Klineberg E, Mundis GM, et al: T1 pelvic angle (TPA) effectively evaluates sagittal deformity and assesses radiographical surgical outcomes longitudinally. Spine (Phila Pa 1976) 39:12031210, 2014

20. Schwab F, Patel A, Ungar B, Farcy JP, Lafage V: Adult spinal deformity-postoperative standing imbalance: how much can you tolerate? An overview of key parameters in assessing 
alignment and planning corrective surgery. Spine (Phila Pa 1976) 35:2224-2231, 2010

21. Schwab FJ, Blondel B, Bess S, Hostin R, Shaffrey CI, Smith JS, et al: Radiographical spinopelvic parameters and disability in the setting of adult spinal deformity: a prospective multicenter analysis. Spine (Phila Pa 1976) 38:E803-E812, 2013

22. Smith JS, Bess S, Shaffrey CI, Burton DC, Hart RA, Hostin $\mathrm{R}$, et al: Dynamic changes of the pelvis and spine are key to predicting postoperative sagittal alignment after pedicle subtraction osteotomy: a critical analysis of preoperative planning techniques. Spine (Phila Pa 1976) 37:845-853, 2012

\section{Disclosures}

Dr. Protopsaltis reports being a consultant for Medicrea, NuVasive, Globus, and Innovasis, and receiving support of non-study- related clinical or research effort from Zimmer Biomet and the Cervical Spine Research Society.

\section{Author Contributions}

Conception and design: Vaynrub, Buckland, Errico, Protopsaltis. Acquisition of data: Vaynrub, Hirsch, Buckland, Protopsaltis. Analysis and interpretation of data: Vaynrub, Tishelman, Buckland, Protopsaltis. Drafting the article: Vaynrub, Tishelman. Critically revising the article: Vaynrub, Hirsch, Tishelman, Buckland, Protopsaltis. Reviewed submitted version of manuscript: all authors. Approved the final version of the manuscript on behalf of all authors: Vaynrub. Statistical analysis: Tishelman, VasquezMontes. Study supervision: Errico, Protopsaltis.

\section{Correspondence}

Max Vaynrub: Hospital for Joint Diseases at NYU Langone Medical Center, New York, NY. max.vaynrub@gmail.com. 